\title{
Stability of the $2+2$ Fermionic System with Point Interactions
}

\author{
Thomas Moser $^{1} \cdot$ Robert Seiringer $^{1}$
}

Received: 26 February 2018 / Accepted: 3 July 2018 / Published online: 23 July 2018 (C) The Author(s) 2018

\begin{abstract}
We give a lower bound on the ground state energy of a system of two fermions of one species interacting with two fermions of another species via point interactions. We show that there is a critical mass ratio $m_{2} \approx 0.58$ such that the system is stable, i.e., the energy is bounded from below, for $m \in\left[m_{2}, m_{2}^{-1}\right]$. So far it was not known whether this $2+2$ system exhibits a stable region at all or whether the formation of four-body bound states causes an unbounded spectrum for all mass ratios, similar to the Thomas effect. Our result gives further evidence for the stability of the more general $N+M$ system.
\end{abstract}

Keywords Point interactions $\cdot$ Stability $\cdot$ Scattering length $\cdot$ Thomas effect

Mathematics Subject Classification (2010) $81 \mathrm{Q} 10 \cdot 46 \mathrm{~N} 50$

\section{Introduction}

Systems of particles interacting via point interactions are frequently used in physics to model short range forces. In these models the shape of the interaction potential

\section{Thomas Moser}

tmoser@ist.ac.at

Robert Seiringer

rseiring@ist.ac.at

1 IST Austria, Am Campus 1, 3400 Klosterneuburg, Austria 
enters only via the scattering length. Originally point interactions were introduced in the 1930s to model nuclear interactions [4, 5, 12, 23, 24], and later they were also successfully applied to other areas of physics like polarons (see [15] and references there) or cold atomic gases [25].

Given $N \geq 1$ fermions of one type with mass $1 / 2$ and $M \geq 1$ fermions of another type with mass $m / 2>0$, point interaction models give a meaning to the formal expression

$$
-\sum_{i=1}^{N} \Delta_{x_{i}}-\frac{1}{m} \sum_{j=1}^{M} \Delta_{y_{j}}+\gamma \sum_{i=1}^{N} \sum_{j=1}^{M} \delta\left(x_{i}-y_{j}\right)
$$

for $\gamma \in \mathbb{R}$. Because of the existence of discontinuous functions in $H^{1}\left(\mathbb{R}^{n}\right)$ for $n \geq$ 2 , this expression is ill-defined in dimensions larger than one. In the following we restrict our attention to the three-dimensional case but we note that the system also exhibits interesting behavior in two dimensions [9, 10, 14].

A mathematically precise version of (1.1) in three dimensions was constructed in $[9,13]$ and we will work here with the model introduced there. We note that even though these models are mathematically well-defined it is not established whether they can be obtained as a limit of genuine Schrödinger operators with interaction potentials of shrinking support. (See, however, [1] for the case $N=M=1$, and [2] for models in one dimension.)

It was already known to Thomas [23] that systems with point interactions are inherently unstable for bosons, in the sense that the energy is not bounded from below, if there are at least three particles involved. It turns out that in the case that the particles are fermions the question of stability is more delicate as it depends on the mass ratio of the two species, in general.

The case $N=M=1$ is completely understood as it reduces to a one particle problem [1]. In this case there exists a one-parameter family of Hamiltonians describing point interactions parameterized by the inverse scattering length, and they are bounded from below for all masses.

Beside this trivial case also the $2+1$ case (i.e., $N=2$ and $M=1$ ), where the two particles of the same species are fermions, is well understood [3, 6-9, 17-20, $22]$. There is a critical mass ratio $m^{*} \approx 0.0735$ such that the system is unstable for $m<m^{*}$ and stable otherwise. It is remarkable that this critical mass ratio does not depend on the strength of the interaction, i.e., the scattering length. Recently in [3] the spectrum of the $2+1$ system was discussed in more detail. Moreover, it was shown in $[7,19]$ that in a certain mass range other models describing point interactions can be constructed.

For larger systems of fermions even the question of stability is generally open. In [21] the stability result for the $2+1$ case was recently extended to the general $N+1$ problem $(N \geq 2$ and $M=1)$. In particular it was shown that there exists a critical mass $m_{1} \approx 0.36$ such that the system's energy is bounded from below, uniformly in $N$, for $m \geq m_{1}$. As a consequence of the $2+1$ case this $N+1$ system is unstable for $m<m^{*}$, but the behavior for $m \in\left[m^{*}, m_{1}\right)$ is unknown.

By separating particles one can obtain an upper bound on the ground state energy of the general $N+M$ problem using the bounds for the $N+1$ or the $1+M$ problem. 
We note that the latter is, up to an overall factor, equivalent to the $M+1$ problem with $m$ replaced by its inverse. Hence the fact that $m_{1}<1$ gives hope that there exists a mass region where the general $N+M$ system is stable for all $N$ and $M$. The simplest problem of this kind is the $2+2$ case. So far there are only numerical results on its stability available $[11,16]$. In particular, the analysis in [11] suggests that the critical mass for the $2+2$ case should be equal to $m^{*}$, i.e., the one for the $2+1$ case.

In this paper we give a rigorous proof of stability for the $2+2$ system in a certain window of mass ratios. We find a critical mass $m_{2} \approx 0.58$ such that the system is stable if $m \in\left[m_{2}, m_{2}^{-1}\right] \approx[0.58,1.73]$. We note that the critical mass $m_{2}$ is not optimal and we cannot make any further statements about the mass range $\left[\mathrm{m}^{*}, m_{2}\right] \cup$ $\left[m_{2}^{-1}, m^{*-1}\right]$. The behavior for these masses, and in particular the question whether $m_{2}=m^{*}$, still represents an open problem.

\section{The Model}

For $p_{1}, p_{2}, k_{1}, k_{2} \in \mathbb{R}^{3}$ and $m>0$, let

$$
h_{0}\left(p_{1}, p_{2}, k_{1}, k_{2}\right)=p_{1}^{2}+p_{2}^{2}+\frac{1}{m}\left(k_{1}^{2}+k_{2}^{2}\right)
$$

We will work with the quadratic form $F_{\alpha}$ introduced in [13] for $2+2$ particles. Its form domain is given by

$$
D\left(F_{\alpha}\right)=\left\{\psi=\varphi+G_{\mu} \xi \mid \varphi \in H_{\mathrm{as}}^{1}\left(\mathbb{R}^{6}\right) \otimes H_{\mathrm{as}}^{1}\left(\mathbb{R}^{6}\right), \xi \in H^{1 / 2}\left(\mathbb{R}^{9}\right)\right\}
$$

where, for some (arbitrary) $\mu>0, G_{\mu} \xi$ is the function with Fourier transform

$$
\widehat{G_{\mu} \xi}\left(p_{1}, p_{2}, k_{1}, k_{2}\right)=\sum_{i, j \in\{1,2\}}(-1)^{i+j}\left(h_{0}\left(p_{1}, p_{2}, k_{1}, k_{2}\right)+\mu\right)^{-1} \hat{\xi}\left(p_{i}+k_{j}, \hat{p}_{i}, \hat{k}_{j}\right)
$$

and we used the notation that $\hat{p}_{1}=p_{2}, \hat{p}_{2}=p_{1}$ and analogously for $k$. The space $H_{\text {as }}^{1}\left(\mathbb{R}^{6}\right)$ denotes antisymmetric functions in $H^{1}\left(\mathbb{R}^{3}\right) \otimes H^{1}\left(\mathbb{R}^{3}\right)$. Note that because of the requirement $\varphi \in H^{1}\left(\mathbb{R}^{12}\right)$ the decomposition $\psi=\varphi+G_{\mu} \xi$ is unique. Note also that the Hilbert space under consideration consists of functions that are antisymmetric in the first two and last two variables, i.e., under both the exchange $p_{1} \leftrightarrow p_{2}$ and $k_{1} \leftrightarrow k_{2}$.

For $\alpha \in \mathbb{R}$, the quadratic form we consider is given by

$$
F_{\alpha}(\psi)=H(\varphi)-\mu\|\psi\|_{2}^{2}+4 T_{\mu}(\xi)+4 \alpha\|\xi\|_{2}^{2},
$$

where

$$
H(\varphi)=\int_{\mathbb{R}^{12}}\left(h_{0}\left(p_{1}, p_{2}, k_{1}, k_{2}\right)+\mu\right)\left|\hat{\varphi}\left(p_{1}, p_{2}, k_{1}, k_{2}\right)\right|^{2} d p_{1} d p_{2} d k_{1} d k_{2}
$$


and $T_{\mu}(\xi)=\sum_{i=0}^{3} \phi_{i}(\xi)$, with the $\phi_{i}$ of the form

$$
\begin{aligned}
& \phi_{0}(\xi)=2 \pi^{2}\left(\frac{m}{m+1}\right)^{3 / 2} \int|\hat{\xi}(P, p, k)|^{2} \sqrt{\frac{P^{2}}{1+m}+p^{2}+\frac{k^{2}}{m}+\mu d P d p d k} \\
& \phi_{1}(\xi)=\int \frac{\hat{\xi}^{*}\left(p_{1}+k_{1}, p_{2}, k_{2}\right) \hat{\xi}\left(p_{2}+k_{1}, p_{1}, k_{2}\right)}{h_{0}\left(p_{1}, p_{2}, k_{1}, k_{2}\right)+\mu} d p_{1} d p_{2} d k_{1} d k_{2} \\
& \phi_{2}(\xi)=\int \frac{\hat{\xi}^{*}\left(p_{1}+k_{1}, p_{2}, k_{2}\right) \hat{\xi}\left(p_{1}+k_{2}, p_{2}, k_{1}\right)}{h_{0}\left(p_{1}, p_{2}, k_{1}, k_{2}\right)+\mu} d p_{1} d p_{2} d k_{1} d k_{2} \\
& \phi_{3}(\xi)=-\int \frac{\hat{\xi}^{*}\left(p_{1}+k_{1}, p_{2}, k_{2}\right) \hat{\xi}\left(p_{2}+k_{2}, p_{1}, k_{1}\right)}{h_{0}\left(p_{1}, p_{2}, k_{1}, k_{2}\right)+\mu} d p_{1} d p_{2} d k_{1} d k_{2}
\end{aligned}
$$

We note that $F_{\alpha}$ is independent of the choice of $\mu>0$. The parameter $\alpha$ corresponds to the inverse scattering length; more precisely, $\alpha=-2 \pi^{2} / a$, with $a \in(-\infty, 0) \cup$ $(0, \infty]$ the scattering length.

It was shown in [13] that $T_{\mu}(\xi)$ is well-defined on $H^{1 / 2}\left(\mathbb{R}^{9}\right)$. To show stability, we need to prove that it is in fact positive. If, on the contrary, there exists a $\mu>0$ and a $\xi \in H^{1 / 2}\left(\mathbb{R}^{9}\right)$ such that $T_{\mu}(\xi)<0$, a simple scaling argument (choosing $\varphi=0$ and using the scale invariance of $F_{0}$ ) can be used to deduce that $F_{\alpha}$ is unbounded from below for all $\alpha \in \mathbb{R}$.

The functionals $\phi_{0}$ and $\phi_{1}$ also appear in a similar form in the discussion of the $2+1$ problem, and $\phi_{2}$ can be seen as the analogous $1+2$ term. The term $\phi_{3}$ has no analogue in the $2+1$ or $1+2$ systems. Note that none of the $\phi_{i}$ for $1 \leq i \leq 3$ has a sign, and we expect that cancellations occur between them that are important for stability. In our proof below, we will first bound $\phi_{0}+\phi_{3}$ from below by a positive quantity, which we then use to compensate separately the negative parts of $\phi_{1}$ and $\phi_{2}$. Since we shall neglect some positive terms, we cannot expect to obtain a sharp bound. In particular, whether $m_{2}=m^{*}$, as suggested in [11], cannot be determined using this method.

\section{Main Result}

For $a \in \mathbb{R}^{3}, b \geq 0$ and $m>0$, let $O_{a, b}^{m}$ be the bounded operator on $L^{2}\left(\mathbb{R}^{3}\right)$ with integral kernel

$$
\begin{aligned}
O_{a, b}^{m}\left(p_{1}, p_{2}\right)= & {\left[\left(p_{1}+a\right)^{2}+b^{2}\right]^{-1 / 4}\left[\left(p_{2}+a\right)^{2}+b^{2}\right]^{-1 / 4} } \\
& \times \frac{1}{p_{1}^{2}+p_{2}^{2}+\frac{2}{1+m} p_{1} \cdot p_{2}+\frac{2(2+m)}{(1+m)^{2}} a^{2}+\frac{2 m}{(1+m)^{2}} b^{2}} .
\end{aligned}
$$

Let further

$$
\Lambda(m)=-\frac{1}{2 \pi^{2}} \frac{1+m}{\sqrt{m}} \inf _{a \in \mathbb{R}^{3}, b \geq 0} \inf \operatorname{spec} O_{a, b}^{m} .
$$


Theorem 1 For $m>0$ such that $\Lambda(m)+\Lambda(1 / m) \leq 1$, we have

$$
T_{\mu}(\xi) \geq(1-\Lambda(m)-\Lambda(1 / m)) \sqrt{2 \mu} \pi^{2}\left(\frac{m}{m+1}\right)^{3 / 2}\|\xi\|_{2}^{2}
$$

for any $\xi \in H^{1 / 2}\left(\mathbb{R}^{9}\right)$ and any $\mu>0$.

This bound readily implies stability for $F_{\alpha}$, as the following corollary shows.

Corollary 1 For $m$ such that $\Lambda(m)+\Lambda(1 / m)<1$, we have

$$
F_{\alpha}(\psi) \geq \begin{cases}0 & \alpha \geq 0 \\ -\alpha^{2}\left(\frac{m+1}{m}\right)^{3} \frac{1}{2 \pi^{4}(1-\Lambda(m)-\Lambda(1 / m))^{2}}\|\psi\|_{2}^{2} & \alpha<0\end{cases}
$$

for any $\psi \in D\left(F_{\alpha}\right)$.

Proof Without loss of generality we can assume that $\|\psi\|_{2}=1$. Using Theorem 1 and $H(\varphi) \geq 0$, we get

$$
\begin{aligned}
F_{\alpha}(\psi)+\mu & \geq 4 T_{\mu}(\xi)+4 \alpha\|\xi\|_{2}^{2} \\
& \geq 4\left[\alpha+(1-\Lambda(m)-\Lambda(1 / m)) \sqrt{2 \mu} \pi^{2}\left(\frac{m}{m+1}\right)^{3 / 2}\right]\|\xi\|_{2}^{2} .
\end{aligned}
$$

In case $\alpha \geq 0$ we obtain $F_{\alpha}(\psi) \geq-\mu$, which shows the result as $\mu>0$ was arbitrary. If $\alpha<0$, we choose

$$
\mu=\alpha^{2}\left(\frac{m+1}{m}\right)^{3} \frac{1}{2 \pi^{4}(1-\Lambda(m)-\Lambda(1 / m))^{2}},
$$

which yields the desired result.

We thus proved stability as long as $\Lambda(m)+\Lambda(1 / m)<1$. To investigate the implication on $m$, let us first check what happens for $a=0$ and $b=0$. An explicit calculation following [6] shows that

$$
\begin{aligned}
\bar{\Lambda}(m) & :=-\frac{1}{2 \pi^{2}} \frac{1+m}{\sqrt{m}} \inf \operatorname{spec} O_{0,0}^{m} \\
& =\frac{2}{\pi}(1+m)^{2}\left(\frac{1}{\sqrt{m}}-\sqrt{2+m} \arcsin \left(\frac{1}{1+m}\right)\right)
\end{aligned}
$$

which satisfies $\bar{\Lambda}(m)+\bar{\Lambda}(1 / m)<1$ for $0.139 \lesssim m \lesssim 7.189$. This range of masses is the largest possible for which our approach can show stability.

While we do not know whether $\Lambda(m)=\bar{\Lambda}(m)$, we shall give in Section 5 a rough upper bound on $\Lambda(m)$ which shows that $\Lambda(m)+\Lambda(1 / m)<1$ for $0.58 \lesssim m \lesssim 1.73$.

\section{Proof of Theorem 1}

We shall split the proof into several steps. 


\subsection{Bound on $\phi_{3}$}

We shall rewrite $\phi_{3}$ in (2.9) using center-of-mass and relative coordinates for each of the pairs $\left(p_{1}, k_{1}\right)$ and $\left(p_{2}, k_{2}\right)$. With $P_{1}=p_{1}+k_{1}, q_{1}=\frac{m}{1+m} p_{1}-\frac{1}{1+m} k_{1}$, $P_{2}=p_{2}+k_{2}$ and $q_{2}=\frac{m}{1+m} p_{2}-\frac{1}{1+m} k_{2}$, we have

$$
\begin{aligned}
\phi_{3}(\xi)=-\int & d P_{1} d P_{2} d q_{1} d q_{2} \\
& \times \frac{\hat{\xi}^{*}\left(P_{1}, \frac{P_{2}}{1+m}+q_{2}, \frac{m P_{2}}{1+m}-q_{2}\right) \hat{\xi}\left(P_{2}, \frac{P_{1}}{1+m}+q_{1}, \frac{m P_{1}}{1+m}-q_{1}\right)}{\frac{1}{1+m}\left(P_{1}^{2}+P_{2}^{2}\right)+\frac{1+m}{m}\left(q_{1}^{2}+q_{2}^{2}\right)+\mu} .
\end{aligned}
$$

By completing the square, we can write, for any positive function $w$,

$$
\begin{aligned}
\phi_{3}(\xi)=\int & \frac{d P_{1} d P_{2} d q_{1} d q_{2}}{w\left(q_{2}, P_{1}, P_{2}\right) w\left(q_{1}, P_{2}, P_{1}\right)} \\
& \times \frac{\frac{1}{2}\left|\chi_{w}\left(q_{2}, P_{1}, P_{2}\right)-\chi_{w}\left(q_{1}, P_{2}, P_{1}\right)\right|^{2}-\left|\chi_{w}\left(q_{2}, P_{1}, P_{2}\right)\right|^{2}}{\frac{1}{1+m}\left(P_{1}^{2}+P_{2}^{2}\right)+\frac{1+m}{m}\left(q_{1}^{2}+q_{2}^{2}\right)+\mu}
\end{aligned}
$$

where we denote $\chi_{w}\left(q, P_{1}, P_{2}\right)=\hat{\xi}\left(P_{1}, \frac{P_{2}}{1+m}+q, \frac{m P_{2}}{1+m}-q\right) w\left(q, P_{1}, P_{2}\right)$. We shall choose

$$
w\left(q, P_{1}, P_{2}\right)=q^{2}+\lambda^{2}\left(\frac{m}{(1+m)^{2}}\left(P_{1}^{2}+P_{2}^{2}\right)+\frac{m}{1+m} \mu\right)
$$

for some constant $\lambda \geq 0$. The first term in the numerator on the right side of (4.2) is manifestly positive. Performing the integration over $q_{1}$, the integral over the second term equals

$$
\begin{aligned}
& \int d P_{1} d P_{2} d q_{2}\left(-\frac{2 \pi^{2} m}{1+m}\right)\left|\hat{\xi}\left(P_{1}, \frac{1}{1+m} P_{2}+q_{2}, \frac{m}{1+m} P_{2}-q_{2}\right)\right|^{2} \\
& \times \frac{q_{2}^{2}+\lambda^{2}\left(\frac{m}{(1+m)^{2}}\left(P_{1}^{2}+P_{2}^{2}\right)+\frac{m}{1+m} \mu\right)}{\lambda \sqrt{\frac{m}{(1+m)^{2}}\left(P_{1}^{2}+P_{2}^{2}\right)+\frac{m}{1+m} \mu}+\sqrt{q_{2}^{2}+\frac{m}{(1+m)^{2}}\left(P_{1}^{2}+P_{2}^{2}\right)+\frac{m}{1+m} \mu}} .
\end{aligned}
$$

Let us compare this latter expression with $\phi_{0}$ in (2.6), which can be rewritten as

$$
\begin{aligned}
& \phi_{0}(\xi)=\frac{2 \pi^{2} m}{m+1} \int\left|\hat{\xi}\left(P_{1}, \frac{1}{1+m} P_{2}+q_{2}, \frac{m}{1+m} P_{2}-q_{2}\right)\right|^{2} \\
& \times \sqrt{q_{2}^{2}+\frac{m}{(1+m)^{2}}\left(P_{1}^{2}+P_{2}^{2}\right)+\frac{m}{1+m} \mu} d P_{1} d P_{2} d q_{2} .
\end{aligned}
$$


For $0 \leq \lambda \leq 1$, one readily checks that

$$
\begin{aligned}
& L_{\lambda}\left(P_{1}, P_{2}, q\right) \\
& :=\sqrt{q^{2}+\frac{m}{(1+m)^{2}}\left(P_{1}^{2}+P_{2}^{2}\right)+\frac{m}{1+m} \mu} \\
& \quad-\frac{q^{2}+\lambda^{2}\left(\frac{m}{(1+m)^{2}}\left(P_{1}^{2}+P_{2}^{2}\right)+\frac{m}{1+m} \mu\right)}{\lambda \sqrt{\frac{m}{(1+m)^{2}}\left(P_{1}^{2}+P_{2}^{2}\right)+\frac{m}{1+m} \mu}+\sqrt{q^{2}+\frac{m}{(1+m)^{2}}\left(P_{1}^{2}+P_{2}^{2}\right)+\frac{m}{1+m} \mu}}
\end{aligned}
$$

is non-negative. What we have shown here is that

$$
\begin{aligned}
& \phi_{0}(\xi)+\phi_{3}(\xi) \\
& \geq \frac{2 \pi^{2} m}{m+1} \int\left|\hat{\xi}\left(P_{1}, \frac{1}{1+m} P_{2}+q, \frac{m}{1+m} P_{2}-q\right)\right|^{2} L_{\lambda}\left(P_{1}, P_{2}, q\right) d P_{1} d P_{2} d q
\end{aligned}
$$

for any $\lambda \geq 0$.

Note that for $\lambda^{2}=1 / 2, L_{\lambda}$ takes the simple form

$$
L_{1 / \sqrt{2}}\left(P_{1}, P_{2}, q\right)=\frac{1}{\sqrt{2}} \sqrt{\frac{m}{(1+m)^{2}}\left(P_{1}^{2}+P_{2}^{2}\right)+\frac{m}{1+m} \mu}
$$

and is, in particular, independent of $q$.

\subsection{Bound on $\phi_{1}$}

For the term $\phi_{1}$ in (2.7), we shall switch to center-of-mass and relative coordinates for the particles $\left(p_{1}, p_{2}, k_{1}\right)$. With $P=p_{1}+p_{2}+k_{1}, q_{1}=\frac{1+m}{2+m} p_{1}-\frac{1}{2+m}\left(p_{2}+k_{1}\right)$ and $q_{2}=\frac{1+m}{2+m} p_{2}-\frac{1}{2+m}\left(p_{1}+k_{1}\right)$, as well as $k=k_{2}$ for short, we have

$$
\begin{aligned}
\phi_{1}(\xi)=\frac{m}{1+m} \int & d P d q_{1} d q_{2} d k \\
& \times \frac{\hat{\xi}^{*}\left(\frac{1+m}{2+m} P-q_{2}, \frac{P}{2+m}+q_{2}, k\right) \hat{\xi}\left(\frac{1+m}{2+m} P-q_{1}, \frac{P}{2+m}+q_{1}, k\right)}{q_{1}^{2}+q_{2}^{2}+\frac{2}{1+m} q_{1} \cdot q_{2}+\frac{m}{(1+m)(2+m)} P^{2}+\frac{1}{1+m} k^{2}+\frac{m}{1+m} \mu} .
\end{aligned}
$$

Defining

$$
\ell_{\lambda}(q, P, k)=L_{\lambda}\left(\frac{1+m}{2+m} P-q, \frac{P}{2+m}+q+k, \frac{m q}{1+m}+\frac{m P}{(1+m)(2+m)}-\frac{k}{1+m}\right)
$$

our aim is to obtain a lower bound on the operator on $L^{2}\left(\mathbb{R}^{3}\right)$ with integral kernel

$$
\frac{\ell_{\lambda}\left(q_{1}, P, k\right)^{-1 / 2} \ell_{\lambda}\left(q_{2}, P, k\right)^{-1 / 2}}{q_{1}^{2}+q_{2}^{2}+\frac{2}{1+m} q_{1} \cdot q_{2}+\frac{m}{(1+m)(2+m)} P^{2}+\frac{1}{1+m} k^{2}+\frac{m}{1+m} \mu}
$$

for suitable $\lambda$, uniformly in the fixed parameters $P$ and $k$. 
Let us take $\lambda^{2}=1 / 2$ for simplicity, in which case we have

$$
\ell_{1 / \sqrt{2}}(q, P, k)=\frac{\sqrt{m}}{1+m} \sqrt{\left(q+\frac{1}{2} k-\frac{m}{2(2+m)} P\right)^{2}+\frac{1}{4}(P+k)^{2}+\frac{1+m}{2} \mu} .
$$

Note also that

$$
\begin{aligned}
& \frac{m}{(1+m)(2+m)} P^{2}+\frac{1}{1+m} k^{2} \\
& =\frac{2 m}{(1+m)^{2}}\left[\frac{2+m}{m}\left(\frac{1}{2} k-\frac{m}{2(2+m)} P\right)^{2}+\frac{1}{4}(P+k)^{2}\right] .
\end{aligned}
$$

With

$$
a=\frac{1}{2} k-\frac{m}{2(2+m)} P \quad, \quad b^{2}=\frac{1}{4}(P+k)^{2}+\frac{1+m}{2} \mu
$$

our task is thus to find a lower bound on the operator with integral kernel $\frac{1+m}{\sqrt{m}} O_{a, b}^{m}\left(q_{1}, q_{2}\right)$, defined in (3.1). The best lower bound equals $-2 \pi^{2} \Lambda(m)$, by definition.

To summarize, what we have shown here is that

$$
\phi_{1}(\xi) \geq-\Lambda(m) \frac{2 \pi^{2} m}{m+1} \int\left|\hat{\xi}\left(\frac{1+m}{2+m} P-q, \frac{P}{2+m}+q, k\right)\right|^{2} \ell_{1 / \sqrt{2}}(q, P, k) d P d q d k \text {. }
$$

Using (4.10), a simple change of variables shows that this is equivalent to

$$
\begin{aligned}
& \phi_{1}(\xi) \\
& \geq-\Lambda(m) \frac{2 \pi^{2} m}{m+1} \int\left|\hat{\xi}\left(P_{1}, \frac{P_{2}}{1+m}+q, \frac{m P_{2}}{1+m}-q\right)\right|^{2} L_{1 / \sqrt{2}}\left(P_{1}, P_{2}, q\right) d P_{1} d P_{2} d q .
\end{aligned}
$$

\subsection{Bound on $\phi_{2}$}

In exactly the same way we proceed with $\phi_{2}$ in (2.8), which we rewrite as

$$
\begin{aligned}
& \phi_{2}(\xi) \\
& =\frac{m}{1+m} \int d P d q_{1} d q_{2} d p \\
& \qquad \times \frac{\hat{\xi}^{*}\left(\frac{1+m}{1+2 m} P-q_{2}, p, q_{2}+\frac{m P}{1+2 m}\right) \hat{\xi}\left(\frac{1+m}{1+2 m} P-q_{1}, p, q_{1}+\frac{m P}{1+2 m}\right)}{q_{1}^{2}+q_{2}^{2}+\frac{2 m}{1+m} q_{1} \cdot q_{2}+\frac{m}{(1+m)(1+2 m)} P^{2}+\frac{m}{1+m} p^{2}+\frac{m}{1+m} \mu} .
\end{aligned}
$$

If we now define

$$
\tilde{\ell}_{\lambda}(q, P, p)=L_{\lambda}\left(\frac{1+m}{1+2 m} P-q, p+q+\frac{m P}{1+2 m}, \frac{m p}{1+m}-\frac{q}{1+m}-\frac{m P}{(1+m)(1+2 m)}\right)
$$

we need a lower bound on the operator on $L^{2}\left(\mathbb{R}^{3}\right)$ with integral kernel

$$
\frac{\tilde{\ell}_{\lambda}\left(q_{1}, P, p\right)^{-1 / 2} \tilde{\ell}_{\lambda}\left(q_{2}, P, p\right)^{-1 / 2}}{q_{1}^{2}+q_{2}^{2}+\frac{2 m}{1+m} q_{1} \cdot q_{2}+\frac{m}{(1+m)(1+2 m)} P^{2}+\frac{m}{1+m} p^{2}+\frac{m}{1+m} \mu}
$$


for fixed $P$ and $p$. By proceeding as in the previous subsection, one readily checks that, for $\lambda^{2}=1 / 2$, its best lower bound is $-2 \pi^{2} \Lambda(1 / m)$, with $\Lambda$ defined in (3.2). In particular, we have

$$
\begin{aligned}
& \phi_{2}(\xi) \\
& \geq-\Lambda(1 / m) \frac{2 \pi^{2} m}{m+1} \int\left|\hat{\xi}\left(P_{1}, \frac{P_{2}}{1+m}+q, \frac{m P_{2}}{1+m}-q\right)\right|^{2} L_{1 / \sqrt{2}}\left(P_{1}, P_{2}, q\right) d P_{1} d P_{2} d q .
\end{aligned}
$$

\subsection{Combining above bounds}

By combining the bounds (4.7), (4.16) and (4.20) from the previous three subsections, we obtain

$$
\begin{aligned}
T_{\mu}(\xi)= & \sum_{j=0}^{3} \phi_{j}(\xi) \\
\geq & (1-\Lambda(m)-\Lambda(1 / m)) \frac{2 \pi^{2} m}{m+1} \\
& \times \int\left|\hat{\xi}\left(P_{1}, \frac{1}{1+m} P_{2}+q, \frac{m}{1+m} P_{2}-q\right)\right|^{2} L_{1 / \sqrt{2}}\left(P_{1}, P_{2}, q\right) d P_{1} d P_{2} d q
\end{aligned}
$$

with $L_{1 / \sqrt{2}}$ defined in (4.8). In the case $\Lambda(m)+\Lambda(1 / m) \leq 1$, we can further use $L_{1 / \sqrt{2}}\left(P_{1}, P_{2}, q\right) \geq \sqrt{m \mu /(2(1+m))}$ for a lower bound. This completes the proof of Theorem 1 .

\section{Bound on $\Lambda(m)$}

Note that $\Lambda(m) \geq \bar{\Lambda}(m)$. To obtain an upper bound, we use the Schur test. We first drop the positive part of the operator with integral kernel

$$
k\left(p_{1}, p_{2}\right)=\left[p_{1}^{2}+p_{2}^{2}+\frac{2}{1+m} p_{1} \cdot p_{2}+\frac{2(2+m)}{(1+m)^{2}} a^{2}+\frac{2 m}{(1+m)^{2}} b^{2}\right]^{-1}
$$

It follows from [21, Lemma 3] that the negative part of this operator has the integral kernel

$$
\begin{aligned}
k_{-}\left(p_{1}, p_{2}\right) & =\frac{-k\left(p_{1}, p_{2}\right)+k\left(p_{1},-p_{2}\right)}{2} \\
& =\frac{2}{1+m} \frac{p_{1} \cdot p_{2}}{\left[p_{1}^{2}+p_{2}^{2}+\frac{2(2+m)}{(1+m)^{2}} a^{2}+\frac{2 m}{(1+m)^{2}} b^{2}\right]^{2}-\frac{4\left(p_{1} \cdot p_{2}\right)^{2}}{(1+m)^{2}}} .
\end{aligned}
$$


By applying the Cauchy-Schwarz inequality, we obtain, for any positive function $h$ on $\mathbb{R}^{3}$ (possibly depending on $a$ and $b$ )

$$
\begin{aligned}
\Lambda(m) \leq \frac{1}{\pi^{2} \sqrt{m}} \sup _{p_{1}, a, b} \int_{\mathbb{R}^{3}} & \frac{h\left(p_{1}\right)}{h\left(p_{2}\right)} \frac{\left|p_{1} \cdot p_{2}\right|}{\left[p_{1}^{2}+p_{2}^{2}+\frac{2(2+m)}{(1+m)^{2}} a^{2}+\frac{2 m}{(1+m)^{2}} b^{2}\right]^{2}-\frac{4\left(p_{1} \cdot p_{2}\right)^{2}}{(1+m)^{2}}} \\
& \times\left[\left(p_{2}+a\right)^{2}+b^{2}\right]^{-1 / 2} d p_{2} .
\end{aligned}
$$

By monotonicity, we can set $b=0$, i.e,

$$
\Lambda(m) \leq \frac{1}{\pi^{2} \sqrt{m}} \sup _{p_{1}, a} \int_{\mathbb{R}^{3}} \frac{h\left(p_{1}\right)}{h\left(p_{2}\right)} \frac{\left|p_{1} \cdot p_{2}\right|}{\left[p_{1}^{2}+p_{2}^{2}+\frac{2(2+m)}{(1+m)^{2}} a^{2}\right]^{2}-\frac{4\left(p_{1} \cdot p_{2}\right)^{2}}{(1+m)^{2}}}\left|p_{2}+a\right|^{-1} d p_{2} .
$$

We shall choose $h$ to be even, i.e., $h(p)=h(-p)$, in which case we can symmetrize to get

$$
\begin{aligned}
& \Lambda(m) \leq \frac{1}{\pi^{2} \sqrt{m}} \sup _{p_{1}, a} \int_{\mathbb{R}^{3}} \frac{h\left(p_{1}\right)}{h\left(p_{2}\right)} \frac{\left|p_{1} \cdot p_{2}\right|}{\left[p_{1}^{2}+p_{2}^{2}+\frac{2(2+m)}{(1+m)^{2}} a^{2}\right]^{2}-\frac{4\left(p_{1} \cdot p_{2}\right)^{2}}{(1+m)^{2}}} \\
& \times \frac{1}{2}\left(\frac{1}{\left|p_{2}+a\right|}+\frac{1}{\left|p_{2}-a\right|}\right) d p_{2} \\
& \leq \frac{1}{\pi^{2} \sqrt{m}} \sup _{p_{1}, a} \int_{\mathbb{R}^{3}} \frac{h\left(p_{1}\right)}{h\left(p_{2}\right)} \frac{\left|p_{1} \cdot p_{2}\right|}{\left[p_{1}^{2}+p_{2}^{2}+\frac{2(2+m)}{(1+m)^{2}} a^{2}\right]^{2}-\frac{4\left(p_{1} \cdot p_{2}\right)^{2}}{(1+m)^{2}}} \\
& \times \sqrt{\frac{p_{2}^{2}+a^{2}}{\left(p_{2}^{2}+a^{2}\right)^{2}-4\left(p_{2} \cdot a\right)^{2}}} d p_{2} .
\end{aligned}
$$

To maximize the right side, $a$ wants to be parallel to $p_{1}$, i.e., $a=\kappa p_{1}$ for $\kappa \in \mathbb{R}$. This is a direct consequence of [21, Lemma 5]. We shall choose $h(p)=|p|$. By scale invariance we can set $\left|p_{1}\right|=1$. We then obtain

$$
\begin{array}{r}
\Lambda(m) \leq \frac{4}{\pi \sqrt{m}} \sup _{\kappa \in \mathbb{R}} \int_{0}^{1} d t \int_{0}^{\infty} d r \frac{r^{2} t}{\left[1+r^{2}+\frac{2(2+m)}{(1+m)^{2}} \kappa^{2}\right]^{2}-\frac{4 r^{2} t^{2}}{(1+m)^{2}}} \\
\times \sqrt{\frac{r^{2}+\kappa^{2}}{\left(r^{2}+\kappa^{2}\right)^{2}-4 \kappa^{2} r^{2} t^{2}}} .
\end{array}
$$

We further bound $t \leq 1$ in the denominator of the first term in the integrand in (5.5), and use that

$$
\left[1+r^{2}+\frac{2(2+m)}{(1+m)^{2}} \kappa^{2}\right]^{2}-\frac{4 r^{2}}{(1+m)^{2}} \geq \frac{m(m+2)}{(1+m)^{2}}\left[1+r^{2}+\frac{2 \sqrt{2+m}}{(1+m) \sqrt{m}} \kappa^{2}\right]^{2} .
$$




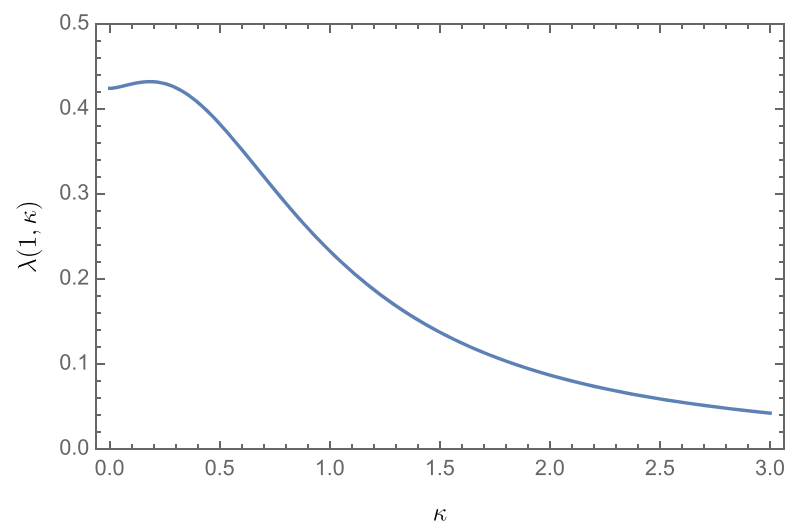

Fig. 1 The function $\lambda(1, \kappa)$, with $\lambda(1)=\sup _{\kappa} \lambda(1, \kappa) \approx 0.427$

Since

$$
\int_{0}^{1} d t t \sqrt{\frac{r^{2}+\kappa^{2}}{\left(r^{2}+\kappa^{2}\right)^{2}-4 \kappa^{2} r^{2} t^{2}}}=\frac{1}{2 r^{2}} \sqrt{r^{2}+\kappa^{2}} \min \left\{1, r^{2} / \kappa^{2}\right\}
$$

we therefore get

$$
\Lambda(m) \leq \frac{2}{\pi} \frac{(1+m)^{2}}{m^{3 / 2}(m+2)} \sup _{\kappa \in \mathbb{R}} \int_{0}^{\infty} d r \frac{\sqrt{r^{2}+\kappa^{2}}}{\left[1+r^{2}+\frac{2 \sqrt{2+m}}{(1+m) \sqrt{m}} \kappa^{2}\right]^{2}}
$$

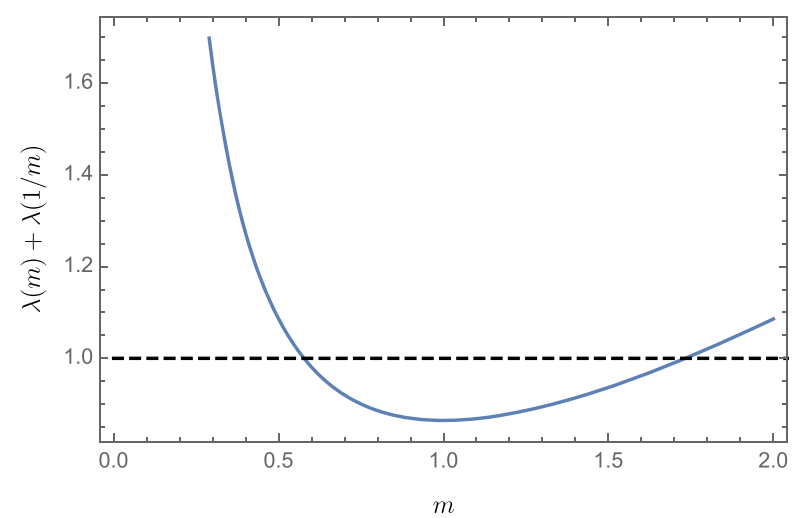

Fig. 2 Our upper bound on $\Lambda(m)+\Lambda(1 / m)$, given by $\lambda(m)+\lambda(1 / m)$ 
We define $c_{m}=2 \sqrt{2+m} /((1+m) \sqrt{m})$. After explicitly doing the integral, the bound (5.8) reads $\Lambda(m) \leq \lambda(m):=\sup _{\kappa>0} \lambda(m, \kappa)$ with

$$
\begin{aligned}
\lambda(m, \kappa):= & \frac{1}{\pi} \frac{(1+m)^{2}}{m^{3 / 2}(m+2)} \frac{1}{1+c_{m} \kappa^{2}}\left(1+\frac{\kappa^{2}}{\sqrt{1+c_{m} \kappa^{2}} \sqrt{1+\kappa^{2}\left(c_{m}-1\right)}}\right. \\
& \left.\times \ln \left(\frac{\sqrt{1+c_{m} \kappa^{2}}+\sqrt{1+\kappa^{2}\left(c_{m}-1\right)}}{\kappa}\right)\right) .
\end{aligned}
$$

For our purpose it is important that $\lambda(1) \approx 0.427<1 / 2$ (see Fig. 1 ). By continuity, this implies that $\Lambda(m)+\Lambda(1 / m)<1$ for a window of mass ratios around 1 . In fact, a numerical optimization over $\kappa$ leads to the conclusion that $\Lambda(m)+\Lambda(1 / m)<$ 1 whenever $0.58 \approx m_{2}<m<m_{2}^{-1} \approx 1.73$ (see Fig. 2).

Acknowledgments Open access funding provided by Austrian Science Fund (FWF).

Open Access This article is distributed under the terms of the Creative Commons Attribution 4.0 International License (http://creativecommons.org/licenses/by/4.0/), which permits unrestricted use, distribution, and reproduction in any medium, provided you give appropriate credit to the original author(s) and the source, provide a link to the Creative Commons license, and indicate if changes were made.

\section{References}

1. Albeverio, S., Gesztesy, F., Høegh-Krohn, R., Holden, H.: Solvable models in quantum mechanics, 2nd edn. American Mathematics Society, Providence (2004)

2. Basti, G., Cacciapuoti, C., Finco, D., Teta, A.: The three-body problem in dimension one: From shortrange to contact interactions, arXiv: 1803.08358

3. Becker, S., Michelangeli, A., Ottolini, A.: Spectral properties of the $2+1$ fermionic trimer with contact interactions, arXiv:1712.10209

4. Bethe, H., Peierls, R.: Quantum theory of the diplon. Proc. R. Soc. Lond. Ser. A 148, 146-156 (1935)

5. Bethe, H., Peierls, R.: The scattering of neutrons by protons. Proc. R. Soc. Lond. Ser. A 149, 176-183 (1935)

6. Correggi, M., Dell'Antonio, G., Finco, D., Michelangeli, A., Teta, A.: Stability for a System of N Fermions plus a different Particle with Zero-Range Interactions. Rev. Math. Phys. 24, 1250017 (2012)

7. Correggi, M., Dell'Antonio, G., Finco, D., Michelangeli, A., Teta, A.: A class of Hamiltonians for a Three-Particle fermionic system at unitarity. Math. Phys. Anal. Geom. 18, 1-36 (2015)

8. Correggi, M., Finco, D., Teta, A.: Energy lower bound for the unitary $N+1$ fermionic model. Eur. Phys. Lett. 111, 10003 (2015)

9. Dell'Antonio, G.F., Figari, R., Teta, A.: Hamiltonians for systems of N particles interacting through point interactions. Ann. Inst. Henri Poincaré, 60, 253-290 (1994)

10. Dimock, J., Rajeev, S.G.: Multi-particle Schrödinger operators with point interactions in the plane. J. Phys. A Math. Gen. 37, 9157-9173 (2004)

11. Endo, S., Castin, Y.: Absence of a four-body Efimov effect in the $2+2$ fermionic problem. Phys. Rev. A 92,053624 (2015)

12. Fermi, E.: Sul moto dei neutroni nelle sostanze idrogenate. Ric. Sci. Progr. Tecn. Econom. Naz. 7, 13-52 (1936)

13. Finco, D., Teta, A.: Remarks on the Hamiltonian for the Fermionic Unitary Gas model. Rep. Math. Phys. 69, 131-159 (2012)

14. Griesemer, M., Linden, U.: Stability of the two-dimensional Fermi polaron. Lett. Math. Phys. 108, 1837-1849 (2018)

15. Massignan, P., Zaccanti, M., Bruun, G.M.: Polarons, dressed molecules and itinerant ferromagnetism in ultracold Fermi gases. Rep. Prog. Phys. 77, 034401 (2014) 
16. Michelangeli, A., Pfeiffer, P.: Stability of the $(2+2)$-fermionic system with zero-range interaction. J. Phys. A: Math. Theor. 49, 105301 (2016)

17. Minlos, R.: On point-like interaction between $n$ fermions and another particle. Mosc. Math. J. 11, 113-127 (2011)

18. Minlos, R.: On pointlike interaction between three particles: Two fermions and another particle. ISRN Math. Phys. 2012, 230245 (2012)

19. Minlos, R.: On pointlike interaction between three particles: Two fermions and another particle II. Mosc. Math. J. 14, 617-637 (2014)

20. Minlos, R.: A system of three quantum particles with point-like interactions. Russ. Math. Surv. 69, 539-564 (2014)

21. Moser, T., Seiringer, R.: Stability of a fermionic $N+1$ particle system with point interactions. Commun. Math. Phys. 356, 329-355 (2017)

22. Shermatov, M.K.: Point Interaction Between Two Fermions and One Particle of a Different Nature. Theor. Math Phys. 136, 1119-1130 (2003)

23. Thomas, L.H.: The interaction between a neutron and a proton and the structure of $H^{3}$. Phys. Rev. 47, 903-909 (1935)

24. Wigner, E.: Über die Streuung von Neutronen an Protonen. Z. Phys. 83, 253-258 (1933)

25. Zwerger, W.: The BCS-BEC Crossover and the Unitary Fermi Gas. Springer Lecture Notes in Physics 836 (2012) 